\title{
Evidence for the flexible use of spatial knowledge in the rat
}

\author{
DOUGLAS B. MATTHEWS and PHILLIP J. BEST \\ Miami University, Oxford, Ohio
}

\begin{abstract}
Animals form spatial representations of environments called cognitive maps, which allow for spatial knowledge to be used in a flexible manner (O'Keefe \& Nadel, 1978). For example, using a cognitive map permits navigation from a novel start location to a known goal location. However, Sutherland, Chew, Baker, and Linggard (1987) showed that in the Morris water maze rats had difficulty finding a known goal location from a novel start location. They concluded that the spatial information contained in the map might not be used in a flexible manner (Sutherland et al., 1987). The present study reveals that the impairment reported by Sutherland et al. is due to a stimulus generalization decrement.
\end{abstract}

Rats construct spatial cognitive maps, or internal representations of spatial locations, for use in organizing behavior (Tolman, 1948) which are thought to be dependent on the hippocampal system (O'Keefe \& Nadel, 1978). According to the cognitive map theory, such maps convey several benefits, including the ability to use spatial information flexibly. This flexibility should permit navigation to a known goal location from a variety of starting locations. Morris (1981) demonstrated that rats trained to swim to a submerged platform in a pool of cloudy water from one start location showed a high degree of positive transfer when they were later started from a novel start location. The degree of transfer was so high that Morris termed it "instantaneous" transfer.

The high degree of transfer on spatial navigation tasks has been demonstrated several times, and has been interpreted as evidence for the use of a cognitive mapping strategy as well as evidence that cognitive mapping is unique in providing such flexible learning (Gallagher \& Holland, 1992; Keith \& McVety, 1988; Morris, Garrud, Rawlins, \& O'Keefe, 1982). However, the phenomenon of positive transfer has been demonstrated in a wide variety of learning paradigms that do not appear to require cognitive mapping. For example, Pavlov (1927) demonstrated that a dog trained to salivate to a tone of a specific frequency as a conditional stimulus (CS) would also salivate to a stimulus that was slightly higher or lower in pitch than the CS, without further training. One principle that Pavlov developed concerning such transfer is that of "stimulus generalization decrement," which holds that when an organism is trained to emit a specific conditioned response (CR) to a particular CS, it will show a greater decrement in CR to stimuli that differ more from

This work was supported in part by a predoctoral award to D.B.M. from the National Institute on Alcohol Abuse and Alcoholism (AA05414 ) and a challenge grant to P.J.B. from the Ohio Board of Regents. Correspondence should be addressed to D. B. Matthews, Center for Alcohol Studies, CB 7178, 3027 Thurston-Bowles Building, Chapel Hill, NC 27599-7178 (e-mail: dougmat@med.unc.edu). the original CS. The more similar the test stimulus is to the original CS, the higher the degree of positive transfer.

In a classical conditioning paradigm, "instantaneous" transfer should occur only if the stimulus conditions during the test are essentially identical to those during the original conditioning. In a similar vein, rats trained to navigate to a submerged platform in the pool of cloudy water would be expected to show "instantaneous" transfer to a novel start location if during early training trials they made frequent excursions in the vicinity of the novel start location. Such an explanation has been offered by Sutherland, Chew, Baker, and Linggard (1987) and Alyan (1994). For example, Sutherland et al. proposed that in Morris's task, rats acquire information about the environment via "incidental viewing" and later use this environmental information during testing. More specifically, Sutherland et al. argued that rats at the beginning of training acquire the information necessary for accurate transfer performance because they tend to swim throughout the entire pool when attempting to find the submerged platform. They concluded that transfer can only be accomplished if the rat is allowed to (1) view all available distal cues and (2) navigate in the vicinity of all segments of the pool early in training (Sutherland et al., 1987, p. 49). Therefore, in the standard Morris paradigm, no location is truly novel at the time of the transfer test. Thus, to test for transfer, one must systematically limit the availability of a portion of the pool to the animal during training, thereby ensuring a novel start location during transfer testing.

Sutherland et al. (1987, Experiment 1) used two salient local cues-a barrier and an opaque curtain-in the pool to restrict the rats' ability to see a subset of cues and to navigate through a portion of the pool during training. When tested from a novel start location, rats were not capable of finding the platform in the fashion described by Morris (1981). However, a review of their methodology can lead to an alternative interpretation. They trained rats to swim to the platform under various experimental conditions (see Sutherland et al., 1987, Figure 1) and then tested the rats from two novel start locations. Some of the con- 
A

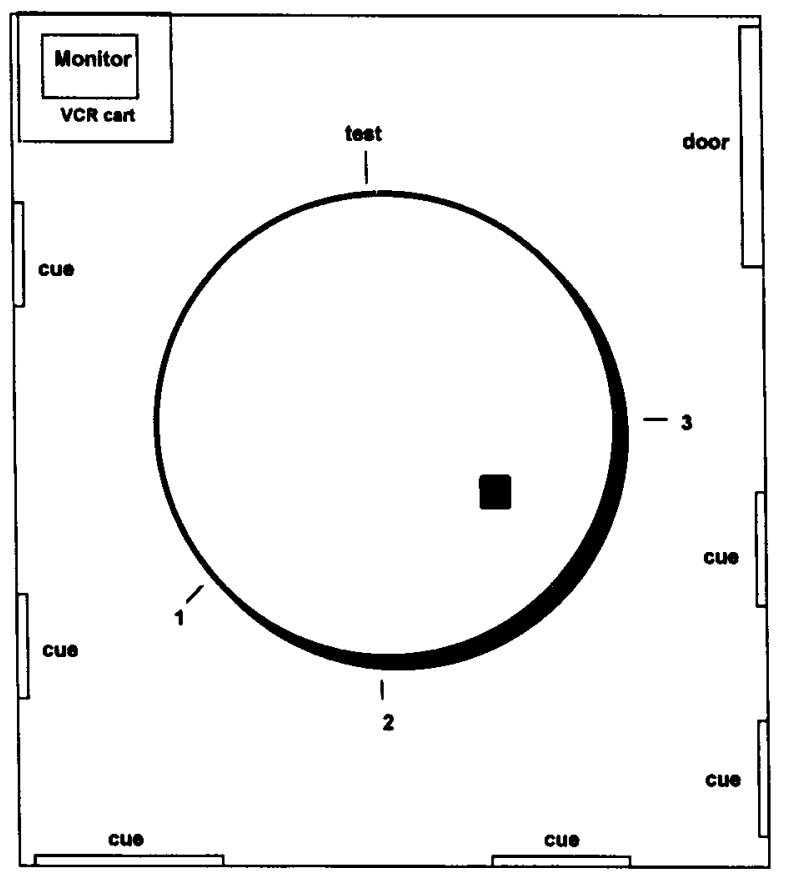

C

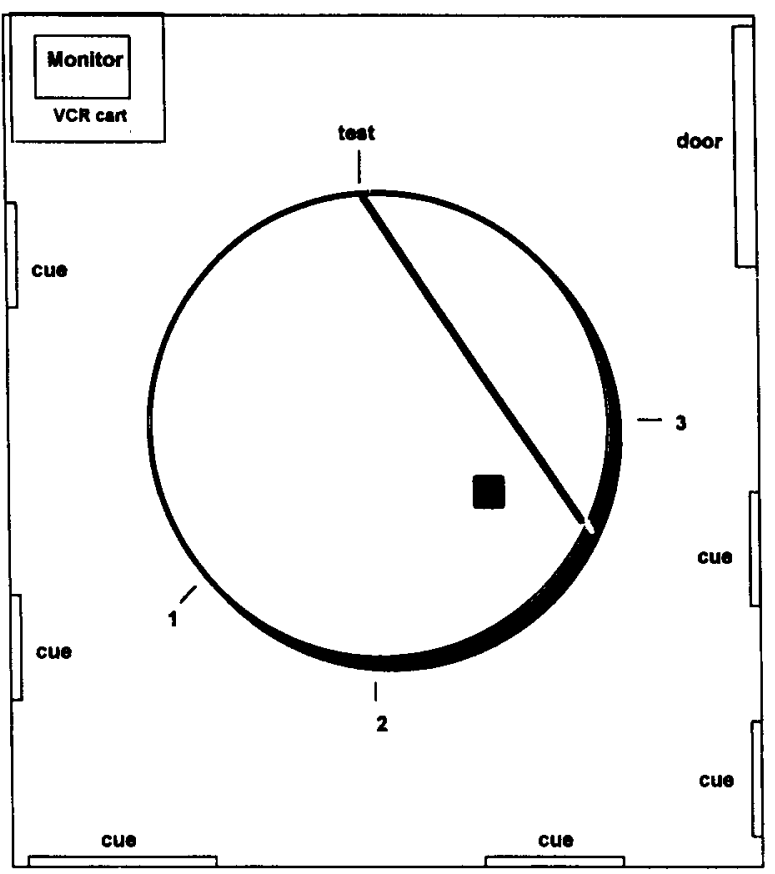

B

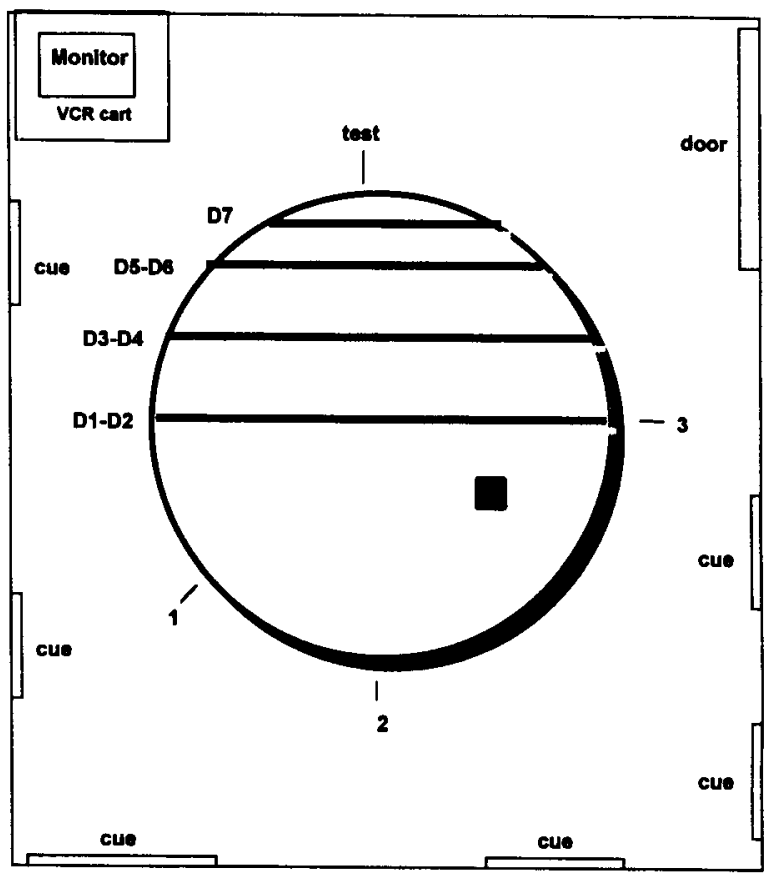

D

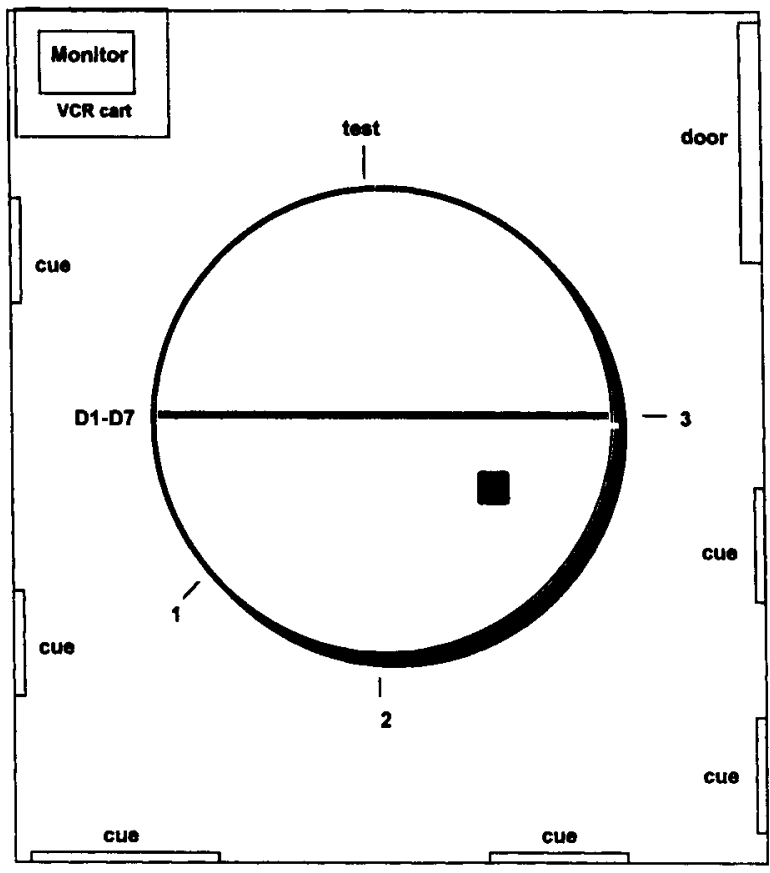

Figure 1. Apparatus during training. The numbers 1-3 denote start locations used during training. "Test" denotes the test start location. The dark square within the maze denotes the placement of the platform. Dark lines within maze denotes location of Plexiglas barrier for each training day. (A) Apparatus for animals in the control condition. (B) Apparatus for animals in the faded barrier condition. (C) Apparatus for animals in the imposed barrier condition. The dark line denotes the placement of the imposed barrier during testing. Note, no barrier was present during training. (D) Apparatus for animals in the removed barrier condition. 
ditions were similar to those of Morris; the rats were permitted to swim through the entire environment and view all of the available cues during training. In the remaining conditions, rats were either restricted to a segment of the pool by a barrier or prevented from viewing all cues by a curtain, or both. During testing, the local cues (i.e., barrier and curtain) were removed and the rats were started from the two novel start locations. The rats trained under the conditions similar to Morris's navigated accurately to the platform during testing. The rats trained under the remaining conditions, however, showed impaired navigation to the platform.

The impairment in performance found in the barriertrained group in Sutherland et al.'s (1987) study may have been due to a stimulus generalization decrement between training and testing. Prior to testing, Sutherland et al. removed what could have been the most salient cues in the pool- the barrier and/or the opaque curtain. Such a deletion of the salient local cues could have disrupted behavior, because even though the animal was familiar with the procedure, the test environment was very different from the training environment.

The present study was designed to test the hypothesis that animals trained to swim to a submerged platform from a set of start locations in the Morris water maze will show a high degree of transfer when tested from a novel start location, even if they have not swum in the vicinity of the novel start location during the original training. A second hypothesis was that a stimulus generalization decrement caused the impairment reported by Sutherland et al. (1987). First, we report that when a barrier bisected the pool during training and was removed prior to the transfer test, animals showed impaired transfer performance, thus replicating Sutherland et al. However, when the barrier was slowly faded during training but still prevented exploration of the novel start location, no impairment in transfer performance was found. Furthermore, animals that were permitted to explore the entire pool during training, including the novel start location, were impaired when a barrier and curtain were imposed into the pool immediately before testing. In other words, removing or adding a barrier just prior to the transfer test caused a significant impairment in transfer performance.

\section{METHOD}

\section{Subjects}

Thirty-seven male rats, 45 days of age, from Charles-River were housed in hanging steel cages on a 12:12-h light:dark cycle (lights on at 7:00 a.m., training between 9:00 a.m. and 4:00 p.m.) in an approved animal colony.

\footnotetext{
Apparatus

All procedures took place in a standard Morris water maze (Morris, 1981), which was approximately $1.8 \mathrm{~m}$ in diameter $\times 0.50 \mathrm{~m}$ high. The pool was located in an experimental room, $2.4 \mathrm{~m} \times 4.25 \mathrm{~m}$, that contained several extra-pool cues. The pool contained a square escape platform $(6.25 \mathrm{~cm} /$ side $\times 38 \mathrm{~cm}$ tall) made of clear Plexiglas that was submerged $2.5 \mathrm{~cm}$ below the water's surface. The es-
}

cape platform could be fitted, via holes drilled in its top, with one of two black wooden caps. The first cap was approximately $5.0 \mathrm{~cm}$ tall, thereby making the platform protrude from the surface by $2.5 \mathrm{~cm}$, while the second cap was approximately $2.5 \mathrm{~cm}$ tall, thereby making the platform flush with the surface. The water in the pool was made cloudy by the addition of white Tempora water color paint. Finally, a clear, $0.50 \mathrm{~m}$ tall $\times 1.8 \mathrm{~m}$ long Plexiglas barrier could be placed along a diameter in the pool, thereby restricting the rats' ability to explore half of the pool. The barrier could be separated into two pieces during training, allowing it to be shortened to approximately $0.9 \mathrm{~m}$.

\section{Behavioral Procedures: Adaptation}

Prior to training, the rats were adapted to the pool, which was located in the center of a cue-restricted chamber. The chamber was constructed of five black curtains hung from a frame suspended from the ceiling. The curtains restricted the rats' ability to see the distal cues in the room for approximately $300^{\circ}$ of visual angle. The final $60^{\circ}$ of visual angle were open, thereby allowing the experimenter access to the pool.

The rats received two adaptation trials from one start location, during which they were trained to swim to the visible platform. The rats were released into the pool facing the wall of the tub, and the swim latency to the visible platform was recorded. If a rat did not find the platform in $60 \mathrm{sec}$, it was gently guided to it. The rats were allowed to remain on the platform for $10 \mathrm{sec}$ before they were removed from the pool and placed in a plastic holding cage that was located on the floor of the test chamber. The intertrial interval was approximately $60 \mathrm{sec}$.

The subjects in the removed-barrier and faded-barrier conditions (described below) were adapted with the clear Plexiglas barrier bisecting the tub. The rats were not allowed to climb onto or over the barrier and therefore could not explore the entire environment.

\section{Training}

The subjects were trained in the same room as the one used in adaptation, except that the curtains were removed to allow them visible access to all available distal cues. During training, the subjects were started from three different start locations twice per day for 7 days and were trained to swim to the platform located in a constant spatial location (see Figure 1A for an example of the pool and the location of the three start locations). The rats were released into the pool facing the wall of the tub, and their swim latency to the platform was recorded. If a rat did not find the platform in $60 \mathrm{sec}$, it was gently guided to it. The rats were allowed to remain on the platform for $10 \mathrm{sec}$ before they were removed from the pool and placed in a plastic holding cage that was located on the floor. The intertrial interval was approximately $90 \mathrm{sec}$. On Training Day 1 , the platform was made visible by using the $5-\mathrm{cm}$ cap; on Training Day 2, the platform was flush to the water surface by using the 2.5-cm cap; and on Training Days 3-8, the platform was submerged $2.5 \mathrm{~cm}$ below the water's surface. Prior to training, the rats were divided into one of four conditions: control $(n=12)$, faded barrier $(n=12)$, removed barrier $(n=6)$, and imposed barrier $(n=7)$. On Training Days 1-7, the subjects in all groups were trained to swim to the platform from the three start locations, twice per day; on the 8th day of training, all subjects were given only one training trial before being tested.

Training procedures for the four conditions are as follows (see Figure 1A-1D for examples):

Subjects in the control condition and the imposed barrier condition were permitted to navigate through and explore all locations of the pool during training.

In the faded barrier condition, the clear Plexiglas barrier was used throughout training to restrict rats' exploration to a selected segment of the pool. On Training Days 1 and 2, the barrier bisected 
the tub, thus restricting the animal from $50 \%$ of the pool. On Training Days 3 and 4, the barrier was moved farther away from the platform, thus restricting the animal from approximately $35 \%$ of the pool. On Training Days 5 and 6, the barrier was moved away once again, thereby restricting the animal from approximately $20 \%$ of the pool. Finally, on Training Day 7 , the barrier was again moved, thereby restricting the animal from $5 \%$ of the pool. The rats were not allowed to climb onto or over the barrier and therefore could not explore the entire environment. This procedure ensured that a segment of the pool (i.e., the test start location) was novel to the animal in terms of both exploration and swim paths.

In the removed barrier condition, the clear Plexiglas barrier was used throughout training to restrict the rats' exploration. On each day of training, the barrier bisected the tub, thereby restricting the rats from $50 \%$ of the pool. The rats were not allowed to climb onto or over the barrier and therefore could not explore the entire environment. This procedure ensured that a segment of the pool (i.e., the test start location) was novel to the animal in terms of both exploration and swim paths. This procedure is very similar to that of Sutherland et al. (1987), Experiment 1, Condition C.

\section{Testing}

On the 8th day of training, immediately following the single training trial, rats were given one test trial in which they were started from the same novel location.

The rats in the control condition were started from the test start location. The rats in the faded barrier condition had the clear Plexiglas barrier shifted clockwise so that one edge was adjacent to the start location before their test trial. The rats in the removed barrier condition had the clear Plexiglas barrier removed before their start trial. The rats in the imposed barrier condition had a barrier and an opaque curtain imposed in the pool in such a manner that the edge of the barrier and curtain were adjacent to the start location before their test trial. However, the barrier and curtain did not block a straight swim trajectory from the start location to the platform.

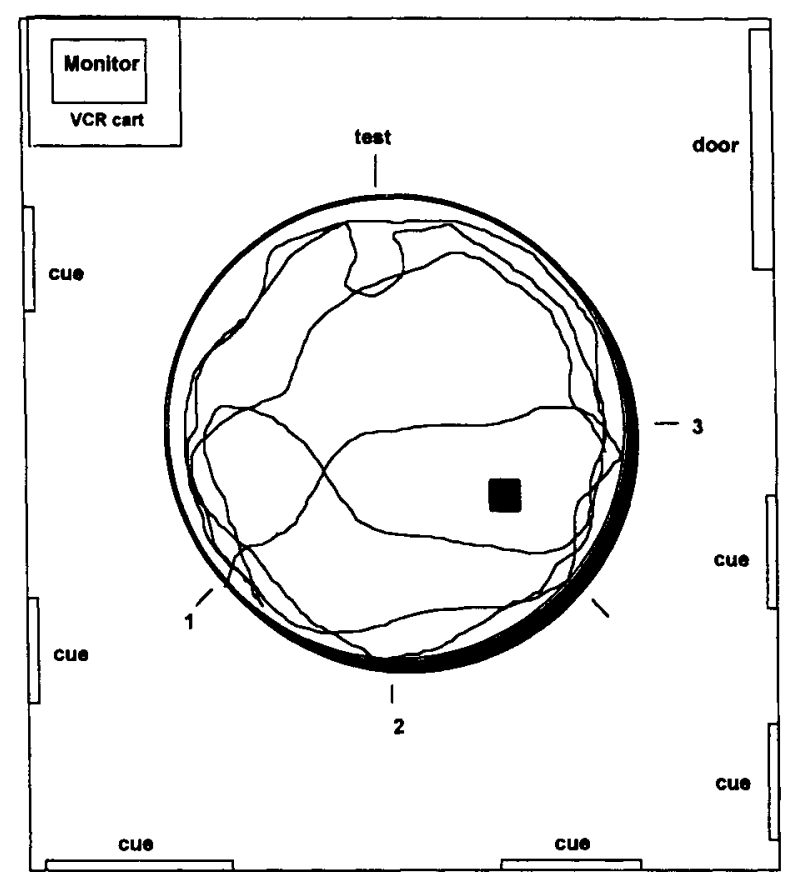

Figure 2. Swim path for a representative animal in the control condition during Day 1 of training. Note that the animal performed similarly to animals in Morris (1981). This line in maze denotes swim path.

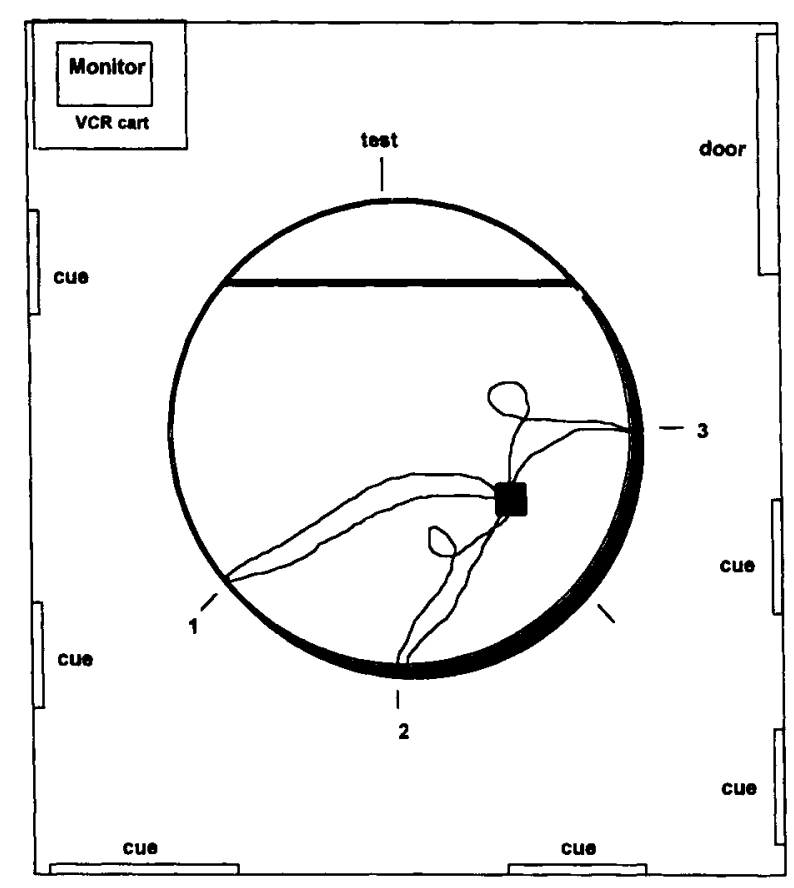

Figure 3. Swim path for a representative animal in the faded barrier condition during Day 6 of training. Note that the animal did not venture to the barrier during training; therefore, that segment of the maze remained unexplored.

\section{RESULTS}

\section{Training}

The rats in all of the conditions demonstrated significant learning of the spatial location of the platform, as was evidenced by their decreased latencies between Day 1 and Day 7 of training (paired $t$ test, all $p$ s <.02). A oneway analysis of variance (ANOVA) showed that performance on the last day of training did not differ significantly between conditions $[F(3,33)=1.67, p>.10]$. Furthermore, rats performed at the same level in the reminder trial on the test day [one-way ANOVA, $F(3,33)=$ $0.75, p>.10]$.

The rats in the control condition behaved similar to Morris's (1981) and Sutherland et al.'s (1987) description of behavior early in training; that is, the rats swam around the edge of the pool, searching for the platform. Thus, the rats in the control condition viewed all available distal cues and navigated in the vicinity of the test start location. See Figure 2 for a representative swim path of a control animal on the 1st day of training. All animals in the faded barrier condition occasionally swam beyond the position where the barrier had been on Training Days 1-2. On Training Days 5, 6, and 7, the animals swam between none and four times beyond the position where the barrier had been on Training Days 3-4. On Day 7 , only 2 of the animals swam beyond the position where the barrier had been on Training Days 5-6. See Figure 3 for the swim paths from an animal in the faded barrier condition on Day 6 of training. 


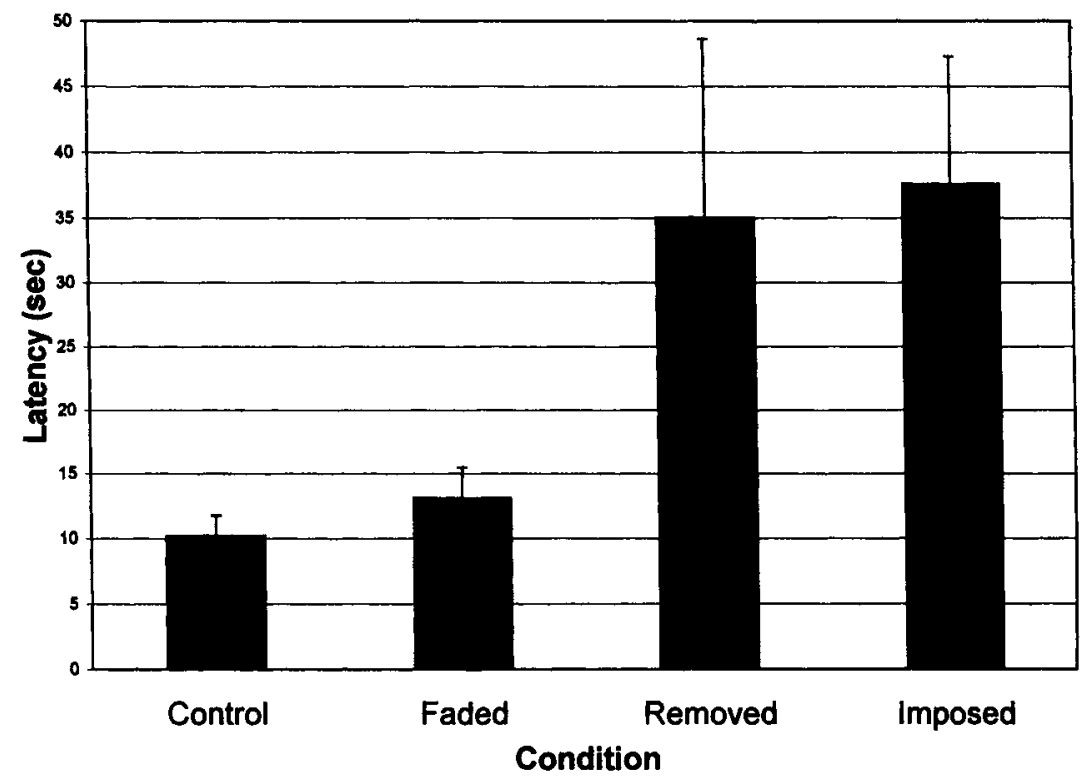

Figure 4. Average swim latencies during the test trial for each condition. Error bars denote the standard error of the mean for each condition.

\section{Testing}

A significant difference between conditions was found on the transfer test day, when the rats were tested from the novel start location [one-way ANOVA, $F(3,33)=5.46$, $p<.01$; see Figure 4]. Comparisons between individual groups, by $t$ tests, revealed that the control and faded barrier conditions did not differ from each other, but that each performed significantly better than the removed barrier and imposed barrier conditions, which also did not differ from each other. Specifically, the rats in the removed barrier condition performed significantly worse than the rats in the control condition [ $t$ test, $T(16)=3.18, p<.02$ ], thus replicating the impairment reported by Sutherland et al. (1987). Performance was also impaired if the barrier was added to the pool before testing, as was evidenced by a significant difference between the imposed barrier and the control conditions [ $t$ test, $T(17)=2.59, p<$ .02]. However, the rats in the faded barrier condition did not differ significantly from rats in the control condition [ $t$ test, $T(22)=1.21, p>.10$ ]. The animals in the removed barrier and the imposed barrier conditions did not significantly differ from each other $[t$ test, $T(11)=0.16, p>$ .10]. However, the animals in both conditions performed significantly worse than animals in the faded barrier condition $[t$ test, $T(16$ and 17$)=2.20$ and 3.10 , respectively, both $p$ s $<.05]$.

See Figures 5-8 for the swim paths of the median animal in each condition during testing.

Since all animals in the faded barrier condition swam beyond previous barrier positions on subsequent training days, additional analyses were performed to determine whether their short latencies on the transfer test day were due to the degree of exploration in the vicinity of the novel start location. If such were the case, we would expect better test transfer in animals who had crossed previous bar- rier positions more frequently or animals who had ventured closer to the novel test start location. That is, we would expect a negative correlation between the number of barrier crossings and latency on the transfer test day, and between the extent of excursions near the transfer test start location and latency on the transfer test day.

Figure 9 shows the latency to reach the platform from the novel start location on the transfer test day as a func-

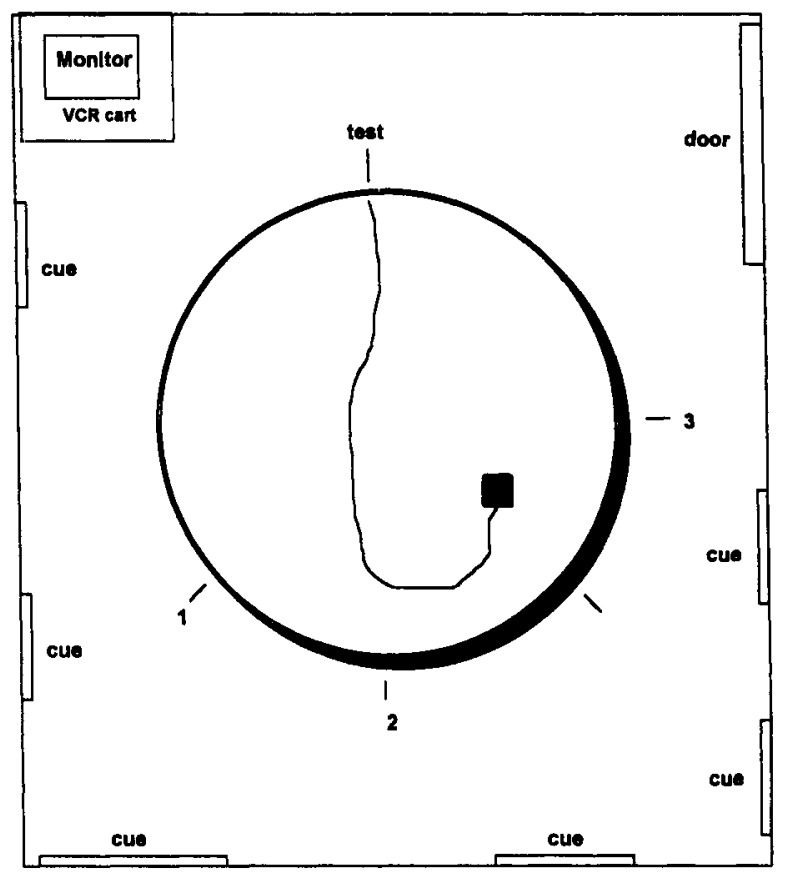

Figure 5. Swim path for the median performer in the control condition test. 


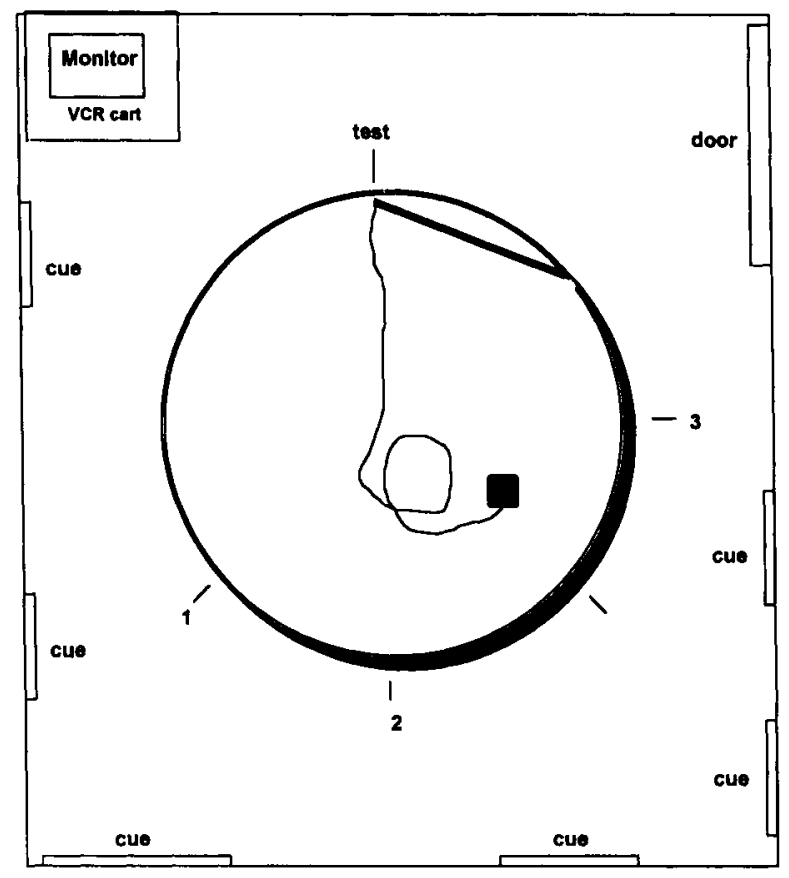

Figure 6. Swim path for the median performer in the faded barrier condition. Dark line in the maze denotes the location of the barrier during testing.

tion of the number of barrier crossings on Training Days 5-7. The figure shows that the latency increases as the number of barrier crossings increases. The correlation between number of barrier crossings and transfer test latency is +.32 (data not shown). If we ignore the crossings of the barrier location for Training Days 1-2, the correlation between the number of times the Days 3-4 and Days 5-6 barrier location was crossed and transfer test latency is +.18 (see Figure 9). To further investigate whether the animals in the faded barrier condition showed transfer because of exploration of the region around the novel start location during training, the animals were divided into two groups. One group of 5 animals either never swam beyond the location of the barrier on Days 3-4 or swam beyond it once, but never swam beyond the location of the barrier on Days 5-6. Their mean latency on the transfer test was $11.1 \mathrm{sec}$. The other group of 7 animals swam beyond the location of the barrier on Days 3-4 from two to six times, and 2 of them also swam beyond the location of the barrier for Days $5-6$. Their mean latency on the transfer test was $14.6 \mathrm{sec}$. Comparison between these two groups reveals that the latency on the transfer test did not differ between the groups [ $t$ test, $t(10)=0.7, p>.10]$. Also note that the animals with less experience near the novel start locations showed a slightly shorter latency - a better performance than did those with more experience near the novel start location.

So that we may further investigate the level of transfer obtained by animals in the faded barrier condition, Figure 10 shows the actual swim paths of a representa- tive animal during Training Days 5-7 and the test trial. Note that this animal never swam within the approximate $33 \%$ of the pool containing the test start location and still displayed good transfer; transfer exhibited by this animal was exactly $27.89 \mathrm{sec}$ better than the mean transfer of animals in the stable barrier condition and $30.43 \mathrm{sec}$ better than the mean transfer of animals in the imposed barrier condition.

\section{DISCUSSION}

The rat's ability to navigate to a known goal location from a novel start location is an important prediction of the spatial cognitive map theory (O'Keefe \& Nadel, 1978, p. 95). This ability has been demonstrated in a number of studies (e.g., Keith \& McVety, 1988; Morris, 1981). However, several studies have questioned this ability (Alyan, 1994; Chew, Sutherland, \& Whishaw, 1989; Sutherland et al., 1987; Whishaw, 1991). Sutherland et al. (1987) have argued that rats must view all available distal cues from the vantage point of the novel start location and must navigate in the vicinity of the novel start location for complete transfer to occur.

The present study provides new data supporting the hypothesis that rats can navigate to a known goal location from a novel start location even when they have never been in the vicinity of the novel start location and are never permitted to view the distal cues from the novel start location during training. Thus, rats can use the information contained within an established spatial cognitive map in a flexible manner. Recall that rats in the faded barrier condition never swam close to the test start loca-

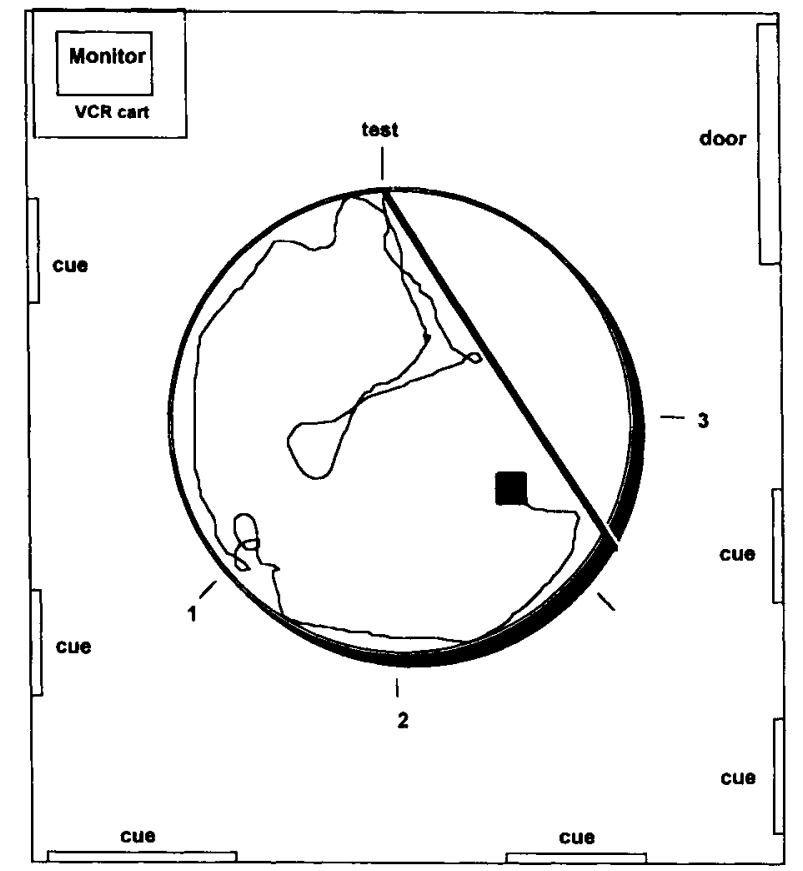

Figure 7. Swim path for the median performer in the imposed barrier condition. 


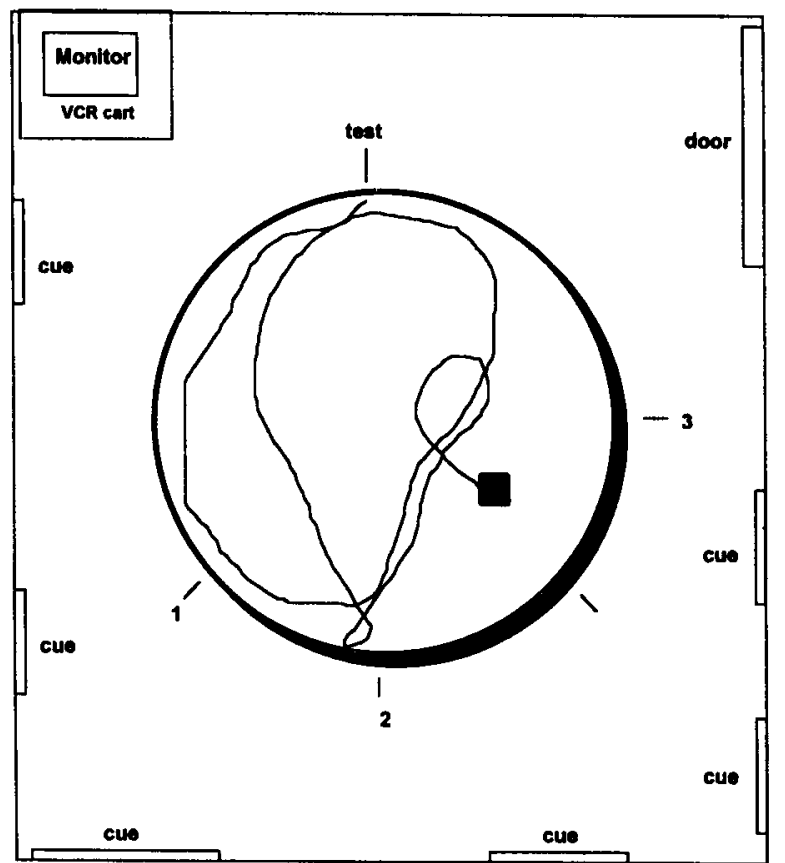

Figure 8. Swim path for the median performer in the removed barrier condition.

tion, and yet they showed the same degree of transfer as did the rats in the control condition.

Sutherland et al. (1987) reported impaired performance when rats were trained under conditions in which their swim path or vision was restricted and were then tested with the barrier and curtain removed from the environ- ment. Although the impaired performance in the restricted animals may have been due to restricting the rats to a segment of the pool, it also could have been due to a stimulus generalization decrement resulting from the major changes in stimulus conditions between training and the transfer test.

The results from the present experiment support the idea that the method used by Sutherland et al. (1987) produced a stimulus generalization decrement. In the current study, rats in both the removed barrier and imposed barrier conditions demonstrated significantly worse performance. In fact, the performance of the rats in the imposed barrier condition was impaired even though they could (1) view all available distal cues during training, (2) navigate in the vicinity (if not through) all portions of the pool during training, and (3) had a straight trajectory to the platform during testing. Thus, the simplest explanation for the deficit in the removed barrier and imposed barrier conditions is that the sudden removal or imposition of the barrier before the test trial caused a significant stimulus generalization decrement.

Sutherland et al.'s (1987) results have been previously reinvestigated by Keith and McVety (1988), who argue that latent learning of the spatial location of a platform in the Morris water pool can lead to facilitated performance on the spatial learning version of the task. However, Chew et al. (1989) argue that Keith and McVety did not demonstrate complete transfer, because all groups showed an increased latency during testing when compared with training, thus demonstrating only partial flexibility of performance. In the present study, the transfer test latency between the control and faded barrier conditions did not significantly differ, and consequently, their

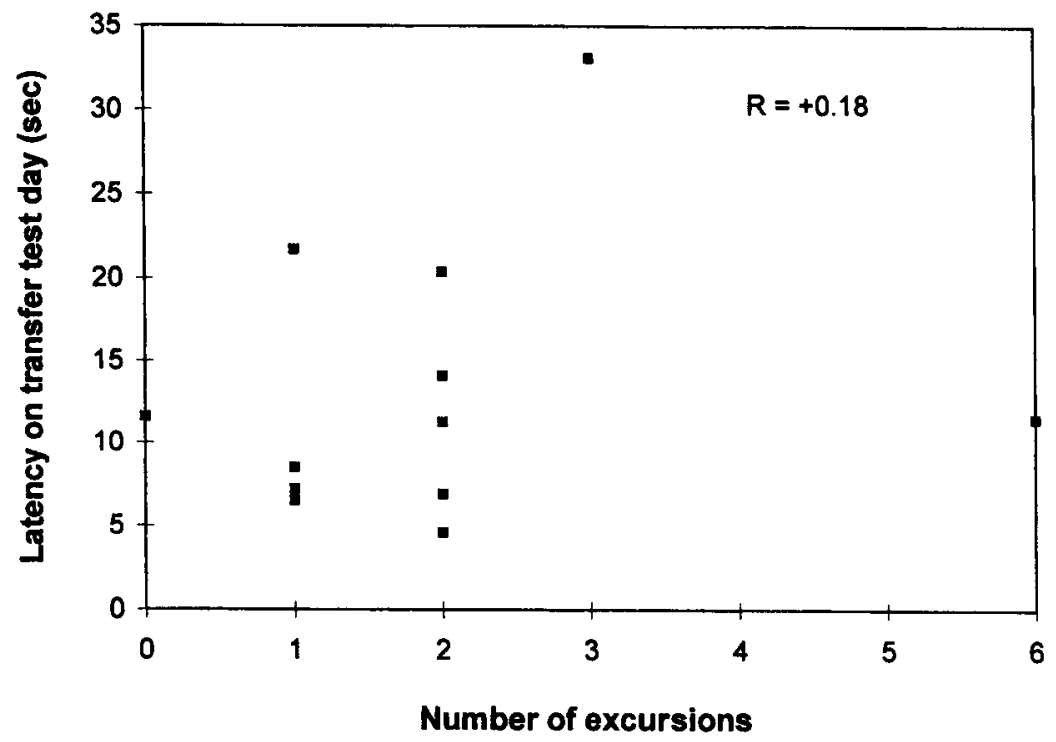

Figure 9. Scatterplot of the number of excursions by animals in the faded barrier condition on Training Days 5-7 into the region of the pool initially restricted by the barrier on Training Days 3-4. The positive correlation shows that the animals which made the fewest excursions into the restricted region of the pool performed the best during the transfer test (i.e., shortest latencies). 
A

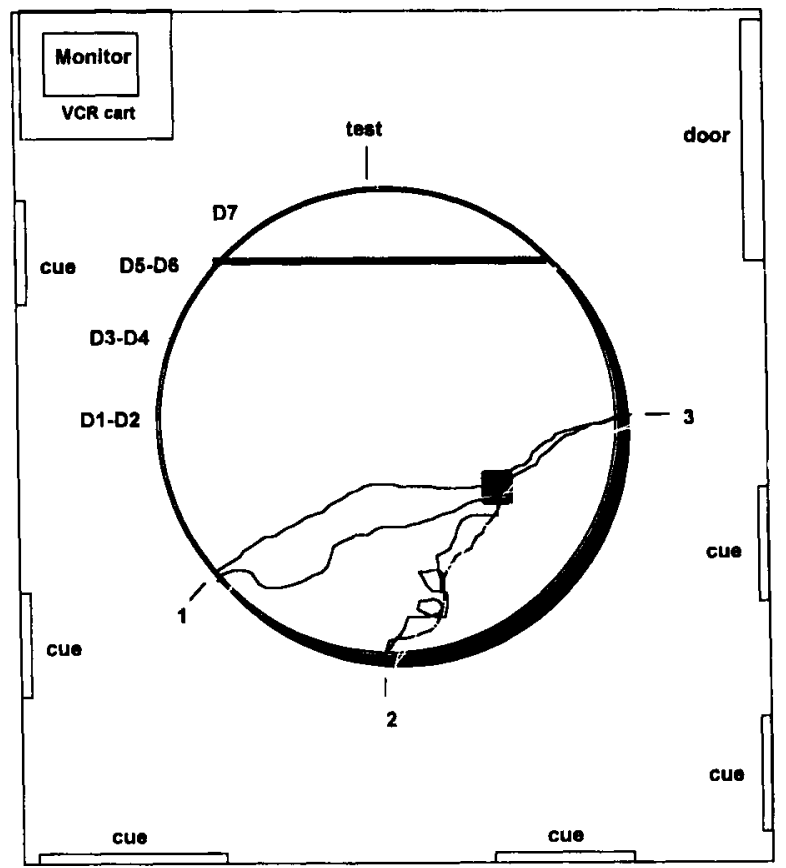

C

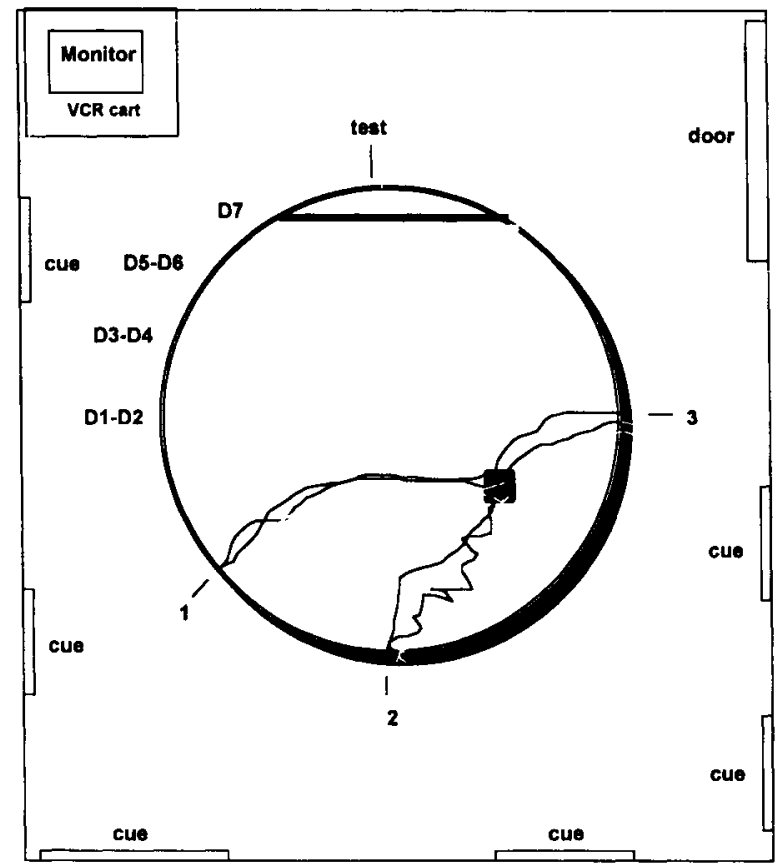

B

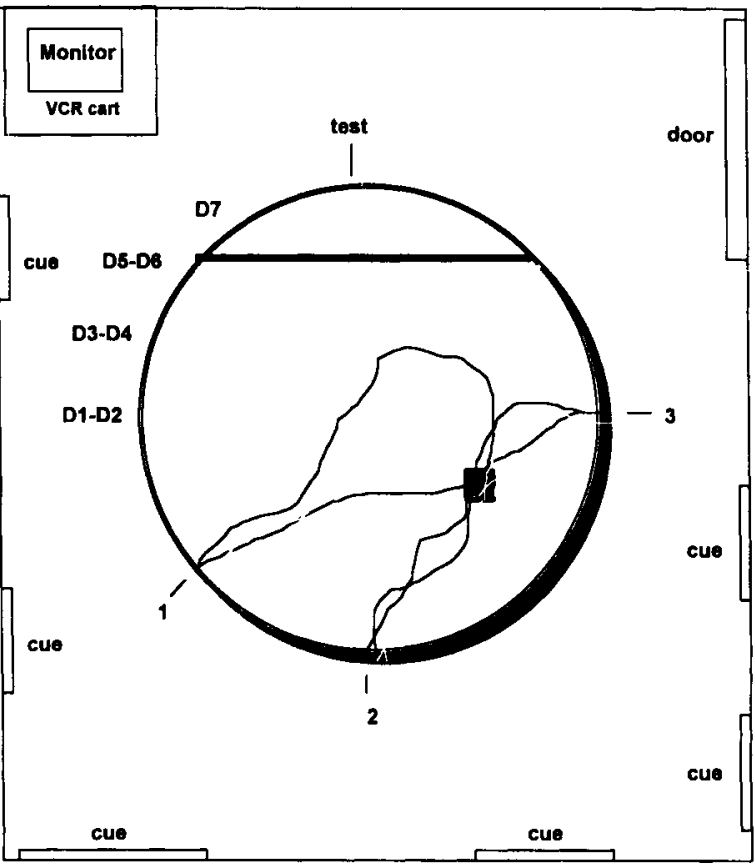

$\mathrm{D}$

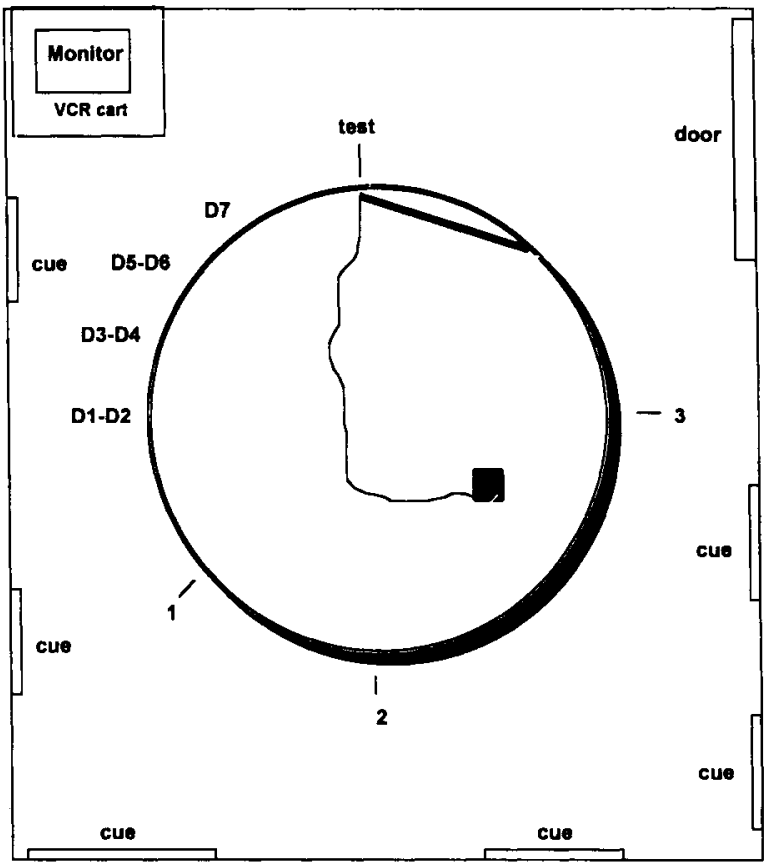

Figure 10: Drawings of the actual swim paths of an animal in the faded barrier condition. The dark line in the pool denotes the location of the barrier; light lines denote the animal's swim paths. (A) Day 5 of training. (B) Day 6 of training. (C) Day 7 of training. (D) Test trial.

latency was essentially identical even though the animals in the faded barrier condition did not ever have the opportunity to explore the novel start location. However, subjects in the removed barrier and imposed barrier conditions had significantly greater latencies than did subjects in the control or faded barrier conditions. The per- formance of the removed barrier and that of the imposed barrier groups also did not significantly differ, and, consequently, their increased latency was essentially identical even though the animals in the imposed barrier condition did have the opportunity to explore the novel start location. Therefore, some cause must have significantly 
impaired the performance of the animals in the removed barrier and imposed barrier conditions. We believe that a stimulus generalization decrement between training and testing due to the addition or removal of salient local cues is the most parsimonious explanation of this data set.

One alternative interpretation of the present results is that rats in the control condition and faded barrier condition are not navigating directly to the platform. Instead, these animals are swimming from the novel portion of the pool to the familiar portion of the pool and then correcting their trajectory. Although that is possible, we believe it to be unlikely for one primary reason. If all an animal had to do was swim to a familiar location and then correct, the animals in the removed barrier and certainly the imposed barrier conditions should perform equally well. However, they clearly do not. Figure 8 shows the typical path taken on the transfer test by the animals in the removed barrier condition. They swam around in circles into and out of the "familiar" region of the pool, almost as if they were not at all familiar with this portion of the pool. We maintain that it was not familiar, but that the removal of the very salient barrier dramatically changed the environment such that it appeared to the animal as a completely novel environment. This argument is supported by the behavior of the animals in the imposed barrier condition, who were never restricted during training, yet showed the same latency as the animals in the removed barrier condition and the same type of swim path (see Figure 7). Thus the removal or imposition of the barrier had the exact same effect on behavior, but restriction had no effect.

A second possible explanation of the effect reported in the present study is that it is, in fact, not contradictory to the results reported by Sutherland et al. (1987) but actually support their results. For example, the difference in performance reported between training on Day 7 and transfer testing in the present study was approximately $5 \mathrm{sec}$ (faded barrier condition), whereas the difference in performance between training and transfer testing reported by Sutherland et al. was also $5 \mathrm{sec}$ (Condition C). Therefore, one could conclude that the animals' performance was the same in the two studies. However, such a conclusion would more than likely be incorrect.

Examination of the data and corresponding figure caption reported in Sutherland et al. (1987, Figure 2) reveals that the performance difference of $5 \mathrm{sec}$ was found by averaging four test trials. In the present study, the 5sec difference was the mean of one test trial. Although it is possible that all four test trials in the Sutherland et al. study resulted in the same latency difference (i.e., $5 \mathrm{sec}$ between training and testing), we do not believe that such is the case. Recall that in the present study, the procedure used in the stable barrier condition was very close to an identical replication of Condition $\mathrm{C}$ in Sutherland et al., yet we report a mean latency difference of approximately $28 \mathrm{sec}$ between training and testing. Further con- founding the issue is the lack of error bars in Figure 2 of the Sutherland et al. study; thus, the range of scores that they recorded during testing cannot be determined. We therefore conclude that the animals in the faded barrier condition were not performing as poorly as the animals in Condition C of Sutherland et al.

One corollary of the spatial cognitive map theory is that animals that are using a mapping strategy should be able to navigate from a novel start location to a known goal location in a given environment (O'Keefe \& Nadel, 1978). The present results provide strong support for this corollary. Animals in the faded barrier condition did not have the opportunity to explore the area surrounding the start location, yet their performance was essentially identical to that of animals in the control condition, which did have the opportunity to explore around the start location. This result is predicted only by the spatial cognitive map theory (O'Keefe \& Nadel, 1978).

The present study supports the finding of transfer of spatial information (Morris, 1981), by showing that rats can accurately navigate to a known goal location from a novel start location. Furthermore, these results are consistent with the spatial cognitive map theory of O'Keefe and Nadel (1978). Finally, the present study provides a reinterpretation of the results of Sutherland et al. (1987) as a stimulus generalization decrement and offers data supporting such a reinterpretation.

\section{REFERENCES}

AlyaN, S. H. (1994). Evidence against instantaneous transfer of spatial knowledge in the house mouse (Mus musculus). Psychobiology, 22, 328-337.

Chew, G. L., Sutherland, R. J., \& Whishaw, I. Q. (1989). Latent learning does not produce instantaneous transfer of place navigation: A rejoinder to Keith and McVety. Psychobiology, 17, 207-209.

Gallagher, M., \& Holland, P. C. (1992). Preserved configural learning and spatial learning impairments in rats with hippocampal damage. Hippocampus, 2, 81-88.

Keith, J. R., \& MCVETY, K. M. (1988). Latent place learning in a novel environment and the influences of prior training in rats. Psychobiology, 16, 146-151

Morris, R. G. M. (1981). Spatial localization does not require the presence of local cues. Learning \& Motivation, 12, 239-260.

Morris, R. G. M., Garrud, P., Rawlins, J. N. P., \& O'Keefe, J. (1982). Place navigation impaired in rats with hippocampal lesions. Nature, 297, 681-683

O'KEEFE, J., \& NADEL, L. (1978). The hippocampus as a cognitive map. Oxford: Oxford University Press.

Pavlov, I. (1927). Conditioned reflexes. (G. V. Anrep, Trans.). London: Oxford University Press.

Sutherland, R. J., Chew, G. L., Baker, J. C., \& Linggard, R. C. (1987). Some limitations on the use of distal cues in place navigation by rats. Psychobiology, 15, 48-57.

Tolman, E. C. (1948). Cognitive maps in rats and men. Psychological Review, 55, 189-208.

Whishaw, I. Q. (1991). Latent learning in a swimming pool place task by rats: Evidence for the use of associative and not cognitive mapping processes. Quarterly Journal of Experimental Psychology, 43B, 83-103.

(Manuscript received August 30, 1996; revision accepted for publication May $30,1997$. ) 